\title{
ANÁLISIS DE GÉNERO EN ESTUDIO DE CASO POR SITUACIONES DE SALUD MENTAL
}

\author{
Erika Alejandra Ramírez Gordillo¹, Maritza Barroso Niño²
}

\section{Resumen:}

Desde 1995, la Organización de Naciones Unidas (ONU, 1995) ha impulsado la estrategia de transversalización del enfoque de género en diversas áreas de política pública. En cuanto el sector salud, la Organización Mundial de la Salud (OMS, 2019) ha integrado dicha estrategia en la búsqueda de la equidad sanitaria en Colombia.

Por lo anterior, en trabajo colaborativo con la Asociación Saludarte (entidad privada sin ánimo de lucro enfocada en la rehabilitación psicosocial de personas con trastorno mental) se realizó la revisión de cuatro casos de personas con trastorno mental, con el fin de identificar a partir de un análisis de género las formas en que las relaciones, normas y funciones de género pudieron estar presentes como factores de riesgo y vulnerabilidad para la ocurrencia de situaciones mórbidas en salud mental. El resultado del análisis en mención se presenta en este artículo como una primera aproximación a la incorporación del análisis de género en los estudios de caso de situaciones de salud mental.

Palabras clave: salud mental, transversalidad de género, trastornos mentales.

Historial del artículo:

Fecha de recibido: 12-04-2021 - Fecha de aceptado: 12-07-2021

(c) 2021 Fundación Universitaria Juan N. Corpas (FUJNC).

Artículo Open Access bajo la Licencia Creative Commons Atribución-NoComercial-

Compartirlgual 4.0 Internacional.

DOI: 10.26752/revistaparadigmassh.v3i1.533

\footnotetext{
${ }^{1}$ Enfermera, epidemióloga, especialista en estudios de género y magíster en Políticas Públicas. Correo electrónico: erikalegordillo@gmail.com

Enfermera especialista en perinatología y coordinadora del Hogar Protegido y Ocupación para Personas con Trastorno Mental (Asociación Saludarte).Autor de correspondencia. Correo electrónico: maritza.barroso@juanncorpas.edu.co
} 


\title{
GENDER ANALYSIS IN A CASE STUDY FOR MENTAL HEALTH SITUATIONS
}

\begin{abstract}
:
Since 1995, the UN has promoted the gender mainstreaming strategy in various areas of public policy. As for the health sector, the World Health Organization (WHO, 2019) has integrated this strategy in the search for health equity and in Colombia.
\end{abstract}

For the above, in collaborative work with the Saludarte Association, a private non- profit entity focused on the psychosocial rehabilitation of people with mental disorder, a review of four (4) cases of people with mental disorder was carried out, in order to identify from a gender analysis, the ways in which gender relations, norms and roles could be present as risk and vulnerability factors for the occurrence of morbid situations in mental health. The result of this analysis is presented in this article as a first approach to the incorporation of gender analysis in case studies of mental health situations.

Keywords: mental health, gender mainstreaming, mental disorders. 


\section{Introducción}

El género es un estructurador social que determina la construcción de roles, valoraciones, estereotipos e imaginarios asociados a lo masculino y lo femenino, y las relaciones de poder que de éstos se desprenden y se exacerban con otras formas de desigualdad. Estas construcciones sociales difieren entre sociedades, culturas y se transforman en el tiempo y parten de expectativas colectivas de género que se modifican dependiendo de la condición de clase, el periodo del curso de vida y el lugar que ocupen los sujetos sociales en el ordenamiento socio-racial. Las discriminaciones por razones de género permean las estructuras sociales, culturales, económicas y políticas; y tienen impactos individuales, comunitarios y colectivos. (Sistema Integrado de Información sobre Violencias de Género [Sivige], 2016, p. 61).

El análisis del género es definido por el Instituto Europeo para la Igualdad de Género (EIGE, por sus siglas en inglés) como "el estudio de las diferencias en las condiciones, necesidades, tasas de participación, acceso a recursos y desarrollo, control de activos, poder para la toma de decisiones, etc., entre mujeres y hombres en los roles de género asignados" (EIGE, 2020). Este instituto resalta que a través del análisis en mención es posible entender inequidades de género en una situación o sector dado, describir las situaciones contextuales y explorar las causas y efectos de las desigualdades de género en grupos específicos.

Un ejemplo de lo anterior son los estudios de caso de género y salud que ha publicado la Organización Panamericana de la Salud, en la que se "[...] analizan los riesgos de hombres y mujeres en relación a enfermedades crónicas, violencia y otros factores. Este análisis tiene como objetivo generar lecciones para la formulación de políticas sensibles al género" (OPS, 2021). En dichos casos, sin embargo, no se identificaron a febrero de 2021 análisis en relación con situaciones de salud mental, en los que el enfoque de género cada vez toma mayor relevancia (Gilligan, 2013).

Por lo anterior, se realizó la revisión de cuatro casos de personas con trastorno mental (Tabla 1), con el fin de identificar por medio de un análisis de género las formas en que las relaciones, normas y papeles según el género pudieron estar presentes como factores de riesgo y vulnerabilidad para la ocurrencia de situaciones mórbidas en salud mental.

El resultado del análisis en mención se presenta en este artículo como una primera aproximación a la incorporación del análisis de género en los estudios de caso de situaciones de salud mental. Por este motivo, el texto se centra en caracterizar aspectos de la relación entre ambas temáticas, organizados en dos entornos (el familiar y el laboral), con el fin de promover su uso tanto por entidades formuladoras de políticas, planes, programas y proyectos, así como por los equipos interdisciplinarios que los implementan, familias, personas con trastornos mentales y organizaciones sociales. No es el objetivo del presente artículo la explicación de cada caso en particular.

El artículo se ordena en cuatro acápites: (I) el análisis de género como herramienta metodológica; (II) reflexiones sobre el entorno familiar; (III) reflexiones sobre el entorno laboral, y (IV) conclusiones.

\section{El análisis de género como herramienta metodológica}

La metodología para la realización del análisis de género, sea sobre una situación o problemática concreta, proyectos o programas, hasta una política, plan o legislación, se consultó en la página web del Instituto Europeo para la Igualdad de Género antes señalado (EIGE 2020). A continuación, se resumen los pasos del análisis y se resaltan los aspectos pertinentes para los estudios de caso que se presentan en este artículo.

Paso 1: recolección de la información. Se enfatiza en la desagregación de datos por sexo y la interseccionalidad con otras formas de discriminación como la edad, la etnicidad, entre otros, así como en la captura de información 
adicional que sea pertinente para verificar temas de género (creencias, imaginarios y demás representaciones sociales, etc.).

Paso 2: identificación de las diferencias de género y las causas subyacentes de las inequidades de género. Implica revelar y examinar diferencias e inequidades en las vidas de hombres y mujeres, al establecer sus causas y efectos. Incluye el análisis de la división sexual del trabajo, los patrones de toma de decisiones, el acceso y control de los recursos sociales, económicos, culturales y políticos, así como las barreras y limitaciones para que los hombres y mujeres participen y se beneficien de manera igualitaria de las políticas, programas o proyectos.

En este paso, es fundamental la selección del marco analítico. En la metodología utilizada por el Instituto Europeo para la Igualdad de Género, se presentan algunas propuestas metodológicas como el marco analítico de Harvard, el marco conceptual de Caroline Moser, el de Levy, el enfoque de capacidades y vulnerabilidades, y el de las relaciones sociales de Naila Kabeer; finalmente, la matriz de análisis de género.

En el presente artículo, se utilizó el marco conceptual de Caroline Moser por su cercanía a las necesidades que atiende el sector salud, tanto de situaciones concretas como de cierre de brechas; es la base del modelo de Moser (Méndez Aguilar, 2010). Dados los distintas funciones, niveles de acceso y control sobre los recursos, las personas tienen distintas necesidades, las cuales Moser (1993) clasificó como prácticas y estratégicas, y señaló que deben tenerse en cuenta al momento de planificar servicios, proyectos, programas, planes o políticas, a manera de interés práctico e interés estratégico, como preocupación o necesidad priorizada en la planificación.

Los intereses prácticos corresponden a las condiciones materiales concretas y necesidades inmediatas en cuanto a las funciones de género que se ejercen; los intereses estratégicos, por su parte, se derivan del análisis de las relaciones de dominio/subordinación. En ambos casos, los intereses de género son definidos como aquellos que mujeres, hombres y personas de los sectores sociales LGBTI pueden desarrollar en virtud de su posición social, la cual, de acuerdo con Moser (1993), es dependiente de una variedad de criterios; entre estos, la clase social, la etnia y el género.

Así, en el presente artículo, se identifican como resultados del análisis de caso las situaciones que evidencian los contextos alienantes para la salud mental, marcados por el género, y que requieren reconocerse como parte de las necesidades prácticas o estratégicas, según corresponda, para satisfacer los intereses antes mencionados. No se centra de la identificación de factores de riesgo o protectores, como habitualmente se habla en el sector salud; si bien las relaciones, normas y funciones de género pueden aplicar en algunos casos como uno u otro según el contexto, la mayoría de las veces estas interceden en factores de riesgo para un buen estado de salud.

En la tabla 1, se presentan algunos datos sociodemográficos no exhaustivos de los casos analizados, los cuales se obtuvieron a partir de la revisión documental de registros de atención en el hogar protegido en mención. 
Tabla 1. Caracterización sociodemográfica de los casos explorados (febrero de 2021).

\begin{tabular}{|c|c|c|c|c|c|c|c|}
\hline Sexo & Edad & Procedencia & Escolaridad & Estrato & NN. de hijos & Diagnóstico & $\begin{array}{c}\text { Años de } \\
\text { diagnóstico }\end{array}$ \\
\hline Femenino & 62 años & Bogotá & $\begin{array}{l}\text { Auxiliar } \\
\text { Contable }\end{array}$ & 3 & 3 & $\begin{array}{c}\text { Trastorno } \\
\text { esquizoafectivo } \\
\text { depresivo }\end{array}$ & 18 años \\
\hline Masculino & 51 años & Medellin & Primaria & 5 & 5 & $\begin{array}{l}\text { Esquizofrenia } \\
\text { paranoide }\end{array}$ & 32 años \\
\hline Femenino & 36 años & Bogotá & Bachillerato & 3 & 3 & $\begin{array}{c}\text { Trastorno } \\
\text { bipolar }\end{array}$ & 10 años \\
\hline Femenino & 66 años & Bogotá & Universitario & 5 & 5 & $\begin{array}{l}\text { Esquizofrenia } \\
\text { paranoide }\end{array}$ & 30 años \\
\hline
\end{tabular}

Fuente: elaboración propia.

\section{Entorno familiar}

El contexto familiar es clave para la promoción de la salud mental y para la prevención de los problemas y trastornos mentales, pues, por un lado, corresponde a un nicho de desarrollo de factores protectores de la individualidad - autoconocimiento, gestión de habilidades y capacidades-; por otro lado, la familia puede participar en la ocurrencia de diversas problemáticas que contribuyen a la presencia de problemas y trastornos en salud mental, así como puede detonar crisis en caso de relaciones interpersonales discriminatorias, conflictivas o de violencia, entre otras.

Uno de los factores que más se resalta en la promoción de la salud mental en el entorno familiar es la construcción de vínculo o apego con la madre o cuidador/a principal. Al tener en cuenta la situación de indefensión del niño/a y el apoyo que requiere la mayor parte del tiempo -más que cualquier otro mamífero-, el/la niño/a depende durante los primeros años de que dicho vínculo y apego le provea lo necesario para su sobrevivencia, entre esto, lo requerido para el desarrollo de una adecuada salud mental. Otro elemento clave en dicho desarrollo mental será el lenguaje (Puche Navarro, 1981), en la construcción de lo simbólico de las relaciones en mención, así como la estructura de estas. Ambos elementos posibilitan en lo biográfico la rehabilitación.

Tanto el vínculo, el apego y el lenguaje resaltan la importancia de los entornos en la promoción de la salud mental, la prevención de los procesos mórbidos, la recuperación y la rehabilitación. Estos factores son influidos por el género, al tener en cuenta que el entorno familiar reproduce per se ordenamientos patriarcales en distintos procesos que se desarrollan en este, como la toma de decisiones, distribución de funciones, reforzamiento de identidades de género hegemónicas y orientación sexual heterosexual, lo que conlleva a distintas formas de desigualdades de género.

El Fondo de Desarrollo de las Naciones Unidas para la Mujer (Unifem) et al. (2010), entidades generadoras de la "Encuesta de Tolerancia Institucional y Social de las Violencias de Género", señalan:

[...] Las relaciones interpersonales más inmediatas y la subjetivación de las experiencias vividas en la interacción con los seres más cercanos intervienen directamente en los comportamientos de los sujetos. (p. 44) 
Estos datos fueron medidos a través de la encuesta citada, mediante diferentes indagaciones asociadas al ordenamiento patriarcal de las familias (Unifem et al., p. 59), entre las que se encuentran:

- Toma de decisiones en cabeza del hombre.

- Control masculino del dinero y los recursos de las mujeres.

- Autoritarismo familiar.

- Sentido de la propiedad, dominación y control sobre las mujeres.

En tres de los cuatro casos analizados, se identificaron factores de riesgo y vulnerabilidad para la salud mental relacionados con el entorno. Los resultados se interpretaron y analizaron de la siguiente forma:

\section{Toma de decisiones en la elección de carrera profesional}

Al tener en cuenta las construcciones generizadas de las carreras profesionales como parte de la división sexual del trabajo, uno de los casos analizados correspondiente a una mujer ingeniera, cuyo padre también lo había sido, resalta la influencia temprana de los estereotipos de género en los intereses infantiles (Bian et al., 2017), los cuales están guiados por los estereotipos de género en relación con lo que "hacen las mujeres" y lo que "hacen los hombres".

Lo anterior refrenda los espacios de la vida privada y pública como femenino y masculino, respectivamente, lo que conlleva al círculo vicioso de "es de mujeres porque ellas desempeñan mejor tal tarea"; entonces, se refuerza que más mujeres se dirijan a ciertas ocupaciones, trabajos y entornos. $\bigcirc$ "es de hombres porque ellos se desempeñan mejor en tal función" y así se impulsa la presencia mayoritaria de hombres. Este proceder desconoce la raíz cultural de tal distribución de funciones, señalada por Bian et al. (2017), así como por Beauvoir (1999), respecto a la socialización generizada de niños y niñas.
Así, si bien la mujer del caso en análisis logra estudiar ingeniería, ella señala que, al iniciar el trabajo como ingeniero con estancia en campamentos, se generaron diversas dificultades para su ejercicio que no se habrían presentado de ser hombre, las cuales se describen como parte del entorno laboral más adelante. En este sentido, quedan interrogantes sobre cómo las expectativas de género que se invisibilizan en la socialización de niños y niñas determinan situaciones futuras laborales, escolares, comunitarias y, a su vez, cómo sesgan la toma de decisiones por hombres, mujeres y personas de los sectores sociales LGBTI en los momentos del curso de la vida.

\section{Violencias de género}

Las violencias de género en el entorno familiar son de las que más se presentan, especialmente la violencia de pareja y las de naturaleza sexual. En uno de los casos analizados, correspondiente a una adulta joven, hija de una mujer con trastorno mental, ambas son víctimas de violencias de género por parte del padre/esposo. La primera sufrió violencia sexual y la segunda, violencia física.

La familia materna, una vez identificada la violencia física contra la madre, le brindó apoyo para vivir lejos del agresor; sin embargo, una vez la hija (joven del estudio de caso), que era adolescente, señaló que el padre debía hacerse cargo de la crianza. No se comprende claramente el hilo de análisis para la toma de la decisión en mención por la familia materna.

Se resalta que: (I) la madre de la joven del estudio de caso no tuvo voz en la decisión que su hija fuera a vivir con su padre, con el cual antes no había vivido, pues la mujer salió del hogar en estado de embarazo; (II) el trastorno mental de la joven se diagnostica posterior a la violencia sexual por parte de su padre, pues la joven refiere que ella, posterior a los hechos, empezó a "sentirse rara, como a no entenderse a sí misma"; (III) pese al proceso judicial del caso de 
violencia sexual, en diversos momentos la joven refiere que se ha desestimado su testimonio "por ser una enferma mental".

\section{Cuerpos masculinos y su "construcción"}

El estudio de caso realizado a un hombre adulto joven con trastorno mental mostró en el relato cómo desde pequeño su padre le instruyó para hacer ejercicio y estimular al máximo el crecimiento muscular, para "ser hombre". El joven señala: "Mire, mi bíceps no se puede tomar con dos manos", lo que en efecto es cierto. Al tener en cuenta las características asignadas a lo masculino en tanto fuerza y destreza física, no es de extrañar el trabajo de performatividad corporal de la fuerza masculina que se muestra en el caso. Se resalta así mismo la admiración femenina, en este caso, de la madre, frente al despliegue de fuerza de su hijo, al referirse a él como "un muchacho muy bien presentado".

A lo anterior, se suma el estímulo para el consumo de alcohol del joven no solo por el padre, sino también por la madre, quien presenta un consumo problemático de alcohol. Este factor y la performatividad en mención hacen parte de la imposición de cánones de masculinidad hegemónica a los niños y jóvenes para llegar a ser "hombres"; aun así, como lo señalan el Unifem et al. (2010), el cuestionamiento a la masculinidad es permanente, por lo cual "ser hombre no es algo dado, es algo en constante demostración" por lo cual la presión referida sobre el joven del caso seguramente se traduciría en la vida adulta en otras presiones para asumir prácticas o funciones del ser "hombre".

\section{Contexto laboral}

Lograr el estatus de trabajador/a supone un estado mental sano, condición inaugural que se mantiene en la medida en que la persona logre mantenerse como trabajador/a. Ahora bien, el supuesto subjetivo de "normalidad del estado mental" que amerita el trabajo dista de las condiciones en que este se ejerce la mayoría de las veces. El Informe Analítico de la Comisión de
Determinantes Sociales de la Salud en el año 2008 refiere una correlación entre tipos de trabajo inestables y el aumento de trastornos mentales (OMS, 2019).

Freud (1929), en el texto El malestar de la cultura, menciona tres aspectos que implican la vida psíquica de los seres humanos y más tarde Cristopher Dejours (2000) lo articula a la situación laboral. Se trata del sufrimiento psíquico inscrito: (a) en la naturaleza del cuerpo; (b) en la exigencia que implica la realidad vs el placer y, (c) las tensiones propias de las relaciones humanas. A continuación, se explican cada una de estas relacionándolas con los estudios de caso:

a. El trabajo se realiza con el cuerpo. El cuerpo de la mujer tiene en el análisis del caso de la mujer ingeniera una presencia determinante. El trabajo tipificado como masculino, que es ejercido por una mujer, impone sobre sus necesidades corporales significaciones avasallantes como:

Espacios comunes de higiene como duchas o lavadero, en los que es posible que la exposición del cuerpo femenino y de ropa interior refuerce chistes sexistas, ocurrencia de violencias y en general el reforzamiento de la cosificación del cuerpo femenino como objeto de deseo sexual.

Discriminación en tanto "las mujeres son complicadas", asociado incluso a necesidades creadas por el mismo sistema de discriminación sexista - por ejemplo, la necesidad de alojamiento y baños aparte que no sería una necesidad si se gozara del derecho de no ser violentado/a y donde su cuerpo no sea cosificado como antes se mencionó.

Las necesidades de privacidad de cualquier cuerpo, en el caso de las mujeres, se sexualizan y descalifican como excluidas de las necesidades humanas que son guiadas especialmente por un canon masculino. Es más fácil denominar como "complicada" o "demasiado exigentes" los 
requerimientos corporales por ser mujer que criticar los condicionamientos que el sistema de discriminación sexista impone a mujeres y hombres. Así, los trabajos estereotipados como ocupaciones de hombres - por ejemplo, la carrera militar, algunas actividades rurales o de construcción-demandan del cuerpo la fuerza, resistencia y capacidad de adaptarse a situaciones que exaltan la fortaleza muscular de por sí performada, principalmente a los hombres, como se expuso en un caso previo, y que excluyen condiciones humanas por considerarse "femeninas".

Un último caso analizado es el relacionado a una mujer auxiliar contable, en quien se desencadenó un trastorno mental en una situación laboral de alta exigencia y sobrecarga. Como se señaló en la tabla 1, la mujer tiene tres hijos/as y por razones de género era quien principalmente ejercía el cuidado de ellos/as. De acuerdo a las encuestas del uso del tiempo elaboradas por el DANE, gestionada a partir de lo ordenado en la Ley 1413 de 2010 "Por la cual se regula la inclusión de la economía del cuidado en el Sistema de Cuentas Nacionales con el objeto de medir la contribución de la mujer al desarrollo económico y social del país y como herramienta fundamental para la definición e implementación de políticas públicas", las mujeres enfrentan dobles jornadas: una de trabajo remunerado y otra de trabajo no remunerado, esta última principalmente con relación a las actividades de cuidado del entorno y de personas en el hogar.

Lo anterior cobra mayor sentido como situación de riesgo en salud mental, cuando se consideran las características de los trabajos domésticos, a saber, invisibles, y que se nota de su existencia cuando no se hacen, impostergables, con grandes exigencias emocionales sobre el/ la cuidadora y muchas veces con dificultad para su delegación, sea por ausencia de personas con quien compartir dicho cuidado o porque quienes están cerca no pueden o no aceptan asumir dicho cuidado. Así, cuando el trabajo re- munerado aumenta las exigencias, sea por necesidades del servicio o por cambios fortuitos, para muchas mujeres con hijos/as les es más difícil adaptarse, lo que genera una tensión de fuerzas en direcciones opuestas que pueden recaer en rupturas, debilitamientos psíquicos y posibles procesos mórbidos.

A lo anterior se suman las representaciones sociales de género que enseñan y exigen de las mujeres que ante una mayor carga laboral, mayor desgaste y posible afectación de su salud mental no se quejen, sino que asuman las nuevas responsabilidades en silencio y lidien con la culpa de no poder responder a los nuevos retos que impone el trabajo o como lo desearían ellas.

b. Hacer frente a lo real vs. placer. Es una apertura al proceso de sublimación por el conocimiento y la creación. Acceder al conocimiento en ciertas ramas de la ciencia ha sido un privilegio masculino y los dos casos que presentamos anteriormente, mujer ingeniera y auxiliar contable, constituyen parte de las excepciones en la posibilidad de acceder a dicha formación. Sin embargo, enfrentarse a la realidad laboral para ellas fue un detonante del trastorno mental, toda vez que es en la práctica que se ponen a prueba las capacidades para transformar la realidad, lo que a su vez exalta las limitaciones diferenciales que los contextos imponen, en este caso, derivadas del género y seguramente en otros sistemas de discriminación como el clasismo, racismo, edadismo, que no se indagaron en el presente estudio.

Transformar la realidad es un proceso creativo e "inventar es también tomar riesgo porque ser ingenioso es con frecuencia estar obligado a hacer excursiones fuera de los procedimientos, es transgredir los reglamentos, es interpretar las órdenes" (Dejours,2000, p. 5), lo cual implica tener un cierto nivel de seguridad y un estado emocional de equilibrio. Y, ¿qué ocurre si se establecen limitaciones discriminantes 
hacia las mujeres? Ellas estarían mucho más expuestas a efectos negativos de la frustración y más presionadas de mantenerse en lugares comunes, que limitarían el desarrollo de su pleno potencial. Entonces, se resaltaría en las frases populares que Ilaman a las mujeres a tener un comportamiento modesto, de bajo perfil ("calladita se ve más bonita") o que no genere conflicto supuesto o verdadero.

Al plantear un análisis interseccional, es importante señalar que lo expuesto no aplica a todas las mujeres por igual, pues no hay una clase social homogénea. Habrá mujeres que al leer estas páginas se reconozcan en sus experiencias, otras que por el contrario no.

c. Se trabaja entablando relaciones. Trabajar hace parte de la relación intrapersonal, interpersonal y social, y resulta muy costoso para el psiquismo un éxito laboral a costa del deterioro de la relación intrapersonal por relaciones que avasallan a la persona. El trabajo es un mediador de la subjetividad sobre el orden social. Dice Dejours (2000) que los vínculos sociales en el trabajo o por el trabajo no son neutros o igualitarios: son relaciones de dominación.

En el año 1998, una publicación de la OlT et al. 2004) alertaba sobre el fenómeno de la violencia en el lugar de trabajo, al aseverar que este constituía una epidemia. A partir de ese momento, una impresionante cantidad de investigaciones sobre el tema comenzaron a emerger, a las que posteriormente se añadieron manuales de actuación, instrumentos de evaluación y normativas. En los casos de las mujeres ingeniera y auxiliar contable, se evidencian relaciones violentas en el entorno laboral y la relación de estas con la ocurrencia de trastornos mentales.

\section{IV.Conclusiones}

El análisis de género en situaciones de salud mental permite evidenciar la correlación entre ambas categorías: el género, con una mirada interseccional de clase social, edad, etnicidad, procedencia, etc., y la salud mental, a partir de una acepción amplia basada en las subjetividades y el psiquismo.

Es fundamental, con base en las reflexiones presentadas, que el análisis de caso que se realice, sea en los niveles operativos comunitarios y clínicos, como en los espacios intersectoriales y sectoriales en los que se formulen políticas, planes, programas, proyectos y servicios, se tenga en cuenta dicha relación de la manera más compleja posible.

Se requiere continuar construyendo conocimiento sobre la relación género y salud mental, con énfasis en entornos específicos, de manera que se contextualice al máximo posible cada conclusión a la que se llegue y así se mantenga un enfoque analítico a nivel macro y micro. Esto se expresa en lo que sigue.

En relación con los casos estudiados, se identifica a la familia como escenario por excelencia para la construcción de una estructura psíquica capaz de adaptarse al desarrollo, al crecimiento y en fin al despliegue del sujeto en el curso de la vida, la cual está permeada por valores y la expresión general de la cultura patriarcal, los cuales tienen un efecto medular en los procesos identitarios de sus integrantes y en el lugar desde donde miran, entienden y viven el mundo. Winnicott (1948) lo llama placement y resalta la capacidad que tiene el entorno de influir en el estado mental de una persona, de tal intimidad que afecta la influencia del ambiente familiar que se instala en la estructura mental. Respecto al entorno laboral, conforme lo expuesto sobre el sufrimiento psíquico a partir 
de las fuentes de sufrimiento planteadas por Freud (1929), las precarias condiciones laborales pueden profundizar el conflicto y desestructurar el funcionamiento mental para el trabajo, esto es: el pensamiento, juicio y raciocinio, toma de decisiones, resolución de problemas, entre otros, que afectan el desempeño del y la trabajador/a y lo /la exponen a la enfermedad mental. Estas condiciones están imbuidas en los sistemas de discriminación sexista, racista, clasista, entre otros, para un todo que no promueve el bienestar del /la trabajador/a.

\section{Referencias Bibliográficas}

1. Beuvoir, S. (1999). El segundo sexo. Editions GaUimard.

2. Bian, L., Leslie, S. J. y Cimpian, A. (2017). Gender stereotypes about intellectual ability emerge early and influence children's interests. Science, 355(6323), 389-391.

https://science.sciencemag.org/content/355/6323/389

3. Dejours, C. (2000). Psicodinámica del trabajo y vínculo social. Actualidad psicológica, (274).

4. Fondo de Desarrollo de las Naciones Unidas para la Mujer (Unifem), Fondo de Población de las Naciones Unidas (Unfpa), Organización Internacional para las Migraciones (OIM), Fondo de las Naciones Unidas y el Gobierno de España para el cumplimiento de los Objetivos de Desarrollo del Milenio (MDGIF). (2010). Encuesta de tolerancia institucional y social a las violencias de género. MDGIF.

5. Freud, S. (1929). El malestar en la cultura.

6. Gilligan, C. (2013). El daño moral y la ética del cuidado. Cuadernos de la Fundación Victor Grifols i Lucas.

7. Instituto Europeo para la Igualdad de Género (EIGE). (2020). Mainstreaming gender: gender analysis. EIGE.

8. Méndez Aguilar, N. (2010). Necesidades prácticas y estratégicas de género. https://www.aciamericas.coop/IMG/pdf/npyegenero.pdf

9. Moser, C. (1993). Gender planning and development -Theory, practice and training (artículo resumen). Routlegde. https://link.springer.com/content/pdf/10.1057\%2Ffr.1995.11.pdf Organización de Naciones Unidas (ONU). (1995). Declaración y Plataforma de Acción de Beijing. ONU. https://bit.ly/3y7nqbE

10. Organización Internacional del Trabajo (OIT), Chappell, D. y Di Martino, V. (2004). La violencia en el trabajo en el sector de servicios y medidas para combatir este fenómeno. Oficina Internacional del Trabajo. 
11. Organización Mundial de la Salud (OMS). (2019). Breaking barriers towards more gender- responsive and equitable Health systems. OMS.

https://www.who.int/healthinfo/universal_health_coverage/report/gender_gm r_2019.pdf?ua=1

12. Organización Panamericana de la Salud (OPS). (2021). Publicaciones sobre género y diversidad cultural: Estudios de caso en género y salud. OPS. https://bit.ly/3yjFWxm

13. Puche Navarro, R. (1981). Lacan: lenguaje e inconsciente. Revista Latinoamericana de Psicología, 3(2), 167-181. https://www.redalyc.org/pdf/805/80503203.pdf

14. Sistema Integrado de Información sobre Violencias de Género (Sivige). (2016). Marco normativo, conceptual y operativo. Ministerio de Justicia, Ministerio de Salud, DANE, Equidad de la Mujer.innicott, D. (1948). Albergues para niños en tiempos de guerra y de paz. Una contribución al simposio sobre "Lessons for Child Psychiatry", leída durante una reunión de la sección médica de la British Psychological Societv.

15. Winnicott, D. (1948). Albergues para niños en tiempos de guerra y de paz. Unacontribución al simposio sobre "Lessons for Child Psychiatry", leída durante una reunión de la sección médica de la British PsychologicalSociety. 